\title{
Fitting a triaxial ellipsoid to a geoid model
}

DOI: https://doi.org/10.1515/jogs-2020-0105

Received March 10, 2020; accepted July 8, 2020

\begin{abstract}
The aim of this work is the determination of the parameters of Earth's triaxiality through a geometric fitting of a triaxial ellipsoid to a set of given points in space, as they are derived from a geoid model. Starting from a Cartesian equation of an ellipsoid in an arbitrary reference system, we develop a transformation of its coefficients into the coordinates of the ellipsoid center, of the three rotation angles and the three ellipsoid semi-axes. Furthermore, we present different mathematical models for some special and degenerate cases of the triaxial ellipsoid. We also present the required mathematical background of the theory of least-squares, under the condition of minimization of the sum of squares of geoid heights. Also, we describe a method for the determination of the foot points of the set of given space points. Then, we prepare suitable data sets and we derive results for various geoid models, which were proposed in the last fifty years. Among the results, we report the semi-axes of the triaxial ellipsoid of geometric fitting with four unknowns to be $6378171.92 \mathrm{~m}, 6378102.06 \mathrm{~m}$ and $6356752.17 \mathrm{~m}$ and the equatorial longitude of the major semi-axis -14.9367 degrees. Also, the parameters of Earth's triaxiality are directly estimated from the spherical harmonic coefficients of degree and order two. Finally, the results indicate that the geoid heights with reference to the triaxial ellipsoid are smaller than those with reference to the oblate spheroid and the improvement in the corresponding rms value is about 20 per cent.
\end{abstract}

Keywords: algebraic fitting, coordinates conversion, geometric fitting, least squares method, principal axes

\section{Introduction}

The problem of determining the parameters of Earth's triaxiality has been recognized since 1859 and various treatments by different scientists have been reported in the

\footnotetext{
*Corresponding Author: G. Panou: Department of Surveying Engineering, National Technical University of Athens, 15780 Athens, Greece, E-mail: geopanou@survey.ntua.gr

R. Korakitis, G. Pantazis: Department of Surveying Engineering, National Technical University of Athens, 15780 Athens, Greece
}

comprehensive list by Heiskanen (1962). Also, recent determinations of the parameters of triaxiality (semi-axes $a_{x}$, $a_{y}$ and $b$, longitude of major semi-axis $\lambda_{0}$ ) are presented in Table 1. However, the difference between the equatorial semi-axes $a_{x}$ and $a_{y}$, as determined in the satellite era, is smaller than the one previously proposed. Some of the reasons have been explained in Burša (1972).

Generally, there are three methods for the determination of the parameters of triaxiality: i) the astrogeodetic, ii) the gravimetric and iii) the satellite method. The gravimetric method is given in detail by Heiskanen (1962). In this method, the parameters of triaxiality are determined using the least-squares method by minimizing the sum of squares of gravity anomalies $\Delta g$. However, in the presatellite era, as mentioned by Heiskanen (1962), the inability to determine the triaxiality parameters with the required accuracy is due to the lack of enough data. The satellite method is described in several papers, e.g. Burša (1970, 1971, 1972) and Burša and Šíma (1980). In this method, the requested parameters are determined using the least-squares method by minimizing the radial distance between a given geoidal point and the corresponding point on the surface of the ellipsoid. On the other hand, once some gravity field model is adopted, from the seconddegree coefficients of spherical harmonics, the orientations of the principal axes and the magnitudes of the principal moments may be determined by a well-known theory (Chen and Shen, 2010).

Nowadays, we can obtain all the data required for applying any of the aforementioned methods. Indeed, there are several satellite-only or combined global gravity field models available by the International Centre for Global Earth Models (ICGEM) (http://icgem.gfzpotsdam.de/home.html). Also, according to Barthelmes (2014), the satellite-only models are computed from satellite measurements alone, whereas ground and altimetry data are additionally used for the combined models. Furthermore, the spatial resolution of the satellite-only models is lower, but the accuracy is nearly uniform over the Earth.

In this work, we determine the parameters of Earth's triaxiality through a least-squares fitting of a triaxial ellipsoid to a set of 'almost equally spaced' geoid points, whose coordinates are of equal precision and are derived 
Table 1. Parameters of Earth's triaxiality in the satellite era

\begin{tabular}{cccccc}
\hline Reference & $a_{x}(\mathrm{~m})$ & $a_{y}(\mathrm{~m})$ & $b(\mathrm{~m})$ & $\lambda_{0}\left({ }^{\circ}\right)$ & $a_{x}-a_{y}(\mathrm{~m})$ \\
\hline Burša and Fialová (1993) & $6378171.36 \pm 0.30$ & $6378101.61 \pm 0.30$ & $6356751.84 \pm 0.30$ & $-14.93 \pm 0.05$ & $69.75 \pm 0.42$ \\
Burša and Šíma (1980) & 6378172 & 6378102.7 & 6356752.6 & -14.9 & 69.3 \\
Eitschberger (1978) & 6378173.435 & 6378103.9 & 6356754.4 & -14.8950 & 69.5 \\
Burša (1972) & 6378173 & 6378104 & 6356754 & -14.8 & 69 \\
Burša (1970) & $6378173 \pm 10$ & $6378105 \pm 18$ & $6356754 \pm 10$ & $-14.8 \pm 5$ & $68 \pm 21$ \\
\hline
\end{tabular}

from a global gravity field model. The condition for the fitting is the minimization of the sum of squares of geoid heights $N$, known as geometric fitting (non-linear), using approximate values for the unknowns derived from an algebraic fitting (linear). Theoretically, the results of geometric fitting are different from those of algebraic fitting and some of the advantages of the geometric fitting are referred to in Bektas (2015). The required mathematical background of the theory of least-squares is included in Mikhail (1976) and Dermanis (2017). Furthermore, in order to illuminate the differences between the triaxial ellipsoid and the oblate spheroid, which has been adopted in geodesy, we consider special and degenerate cases of the triaxial ellipsoid in the fitting. For this scope, we start from a Cartesian equation of an ellipsoid in an arbitrary reference system, as given in Hirvonen (1971), and we develop a transformation of the unknowns of the fitting into parameters, following the algorithm of determining an error ellipsoid from a $3 \times 3$ variance-covariance matrix (for the details see Hirvonen (1971)). Taking into account the improvement of the global gravity field models in the last fifty years, we also derive results for several past geoid models. Finally, we compare the results with those directly derived from the coefficients of a global gravity field model.

The main contributions of the present work can be summarized as follows:

- it offers updated values for the triaxial parameters of the Earth, derived from a large volume of contemporary data, filling the gap in the relevant geodetic literature since the seventies, which was mostly due to the use of the oblate spheroid as the adopted reference surface. Nowadays, however, the accuracy requirements are higher and the computing capabilities are greatly improved.

- starting from a Cartesian equation of an ellipsoid in an arbitrary reference system, we develop a transformation of its polynomial coefficients into geometrical parameters, like the coordinates of the ellipsoid center, the three rotation angles and the three ellipsoid semi-axes. Furthermore, we present different mathematical models for some special and degenerate cases of the triaxial ellipsoid, including the oblate spheroid and the sphere.

- the problem of geometric fitting is solved following an iterative approach, as a least-squares problem with constraints involving additional parameters and using a very large data set. In contrast, Bektas (2015) uses the Levenberg-Marquardt algorithm for solving the non-linear least-squares problem of geometric fitting, in order to determine its parameters. In addition, he uses a general algorithm for the determination of the distance between a data point and its closest point on the ellipsoid, as presented in Bektas (2014). Finally, as an application of his method, he presents a numerical example for a virtual body using only 12 points.

- the presented procedure can be also used for a geometric fitting of the physical surface of the Earth, as mentioned in Tserklevych, Zaiats and Shylo (2016), as well as of the physical surfaces of other celestial bodies, as presented in Karimi, Ardalan and Farahani (2016, 2017), who use an algebraic fitting in their work. This capability is of special importance for other celestial bodies, where the lack of gravity field models does not permit the estimation of their triaxial parameters.

- it vividly demonstrates and verifies the numerical stability of the values of the triaxial parameters of the Earth. As pointed out in Heiskanen (1962), the triaxiality is geophysically interesting and important. For example, it is important in modifying the Earth rotation theory, since it is closely related to the principal moments of inertia of the Earth (Chen and Shen, 2010). Hence, this task should be a challenge for the following years. 


\section{Cartesian equation of an ellipsoid in an arbitrary reference system}

The Cartesian equation of an ellipsoid, in an arbitrary reference system $x y z$, may be given by (Hirvonen 1971)

$f(x, y, z) \doteq\left[\begin{array}{lll}x-t_{x} & y-t_{y} & z-t_{z}\end{array}\right]\left[\begin{array}{lll}q_{x x} & q_{x y} & q_{x z} \\ q_{x y} & q_{y y} & q_{y z} \\ q_{x z} & q_{y z} & q_{z z}\end{array}\right]^{-1}$
$\left[\begin{array}{l}x-t_{x} \\ y-t_{y} \\ z-t_{z}\end{array}\right]=1$

where the elements of vector

$$
\mathbf{t}=\left[\begin{array}{l}
t_{x} \\
t_{y} \\
t_{z}
\end{array}\right]
$$

are the coordinates of the ellipsoid center and the elements of symmetric matrix

$$
\mathbf{Q}=\left[\begin{array}{ccc}
q_{x x} & q_{x y} & q_{x z} \\
& q_{y y} & q_{y z} \\
\text { sym. } & & q_{z z}
\end{array}\right]
$$

are functions of the three rotation angles $\theta_{x}, \theta_{y}$ and $\theta_{z}$, and the three ellipsoid semi-axes $a_{x}, a_{y}$ and $b$ (see subsection 2.4). Thus, the mathematical model given by Eq. (1), includes nine parameters corresponding to the spatial properties of an ellipsoid (translation, orientation, semiaxes). Also, we can consider different cases of an ellipsoid (T1 - S4), as presented in Table 2. In this table, the symbol " $x$ " refers to a parameter that will be determined, whereas the symbol " 0 " indicates that the corresponding parameter is not included in the mathematical model. Thus, apart from the general case (T1) of a triaxial ellipsoid, we consider some special cases (T2-T6), two cases (B3 and B4) of an oblate spheroid without rotations and two cases (S3 and S4) of a sphere.

For each of the above mentioned cases, we present the corresponding mathematical model, as follows.

\subsection{Triaxial ellipsoid and special cases}

\subsubsection{General case T1}

Equation (1) is equivalently written as

$$
\begin{aligned}
& {\left[\begin{array}{lll}
x-t_{x} & y-t_{y} & z-t_{z}
\end{array}\right]\left[\begin{array}{lll}
p_{x x} & p_{x y} & p_{x z} \\
p_{x y} & p_{y y} & p_{y z} \\
p_{x z} & p_{y z} & p_{z z}
\end{array}\right]} \\
& {\left[\begin{array}{l}
x-t_{x} \\
y-t_{y} \\
z-t_{z}
\end{array}\right]=1}
\end{aligned}
$$

or

$\frac{p_{x x}}{d} x^{2}+\frac{p_{y y}}{d} y^{2}+\frac{p_{z z}}{d} z^{2}+\frac{2 p_{x y}}{d} x y+\frac{2 p_{x z}}{d} x z+\frac{2 p_{y z}}{d} y z$

$-\frac{2}{d}\left(p_{x x} t_{x}+p_{x y} t_{y}+p_{x z} t_{z}\right) x-\frac{2}{d}\left(p_{x y} t_{x}+p_{y y} t_{y}+p_{y z} t_{z}\right) y$

$-\frac{2}{d}\left(p_{x z} t_{x}+p_{y z} t_{y}+p_{z z} t_{z}\right) z-1=0$

where

$d=1-p_{x x} t_{x}^{2}-p_{y y} t_{y}^{2}-p_{z z} t_{z}^{2}-2 p_{x y} t_{x} t_{y}-2 p_{x z} t_{x} t_{z}-2 p_{y z} t_{y} t_{z}$

Equation (5) is of the form

$c_{x x} x^{2}+c_{y y} y^{2}+c_{z z} z^{2}+c_{x y} x y+c_{x z} x z+c_{y z} y z+c_{x} x+c_{y} y+c_{z} z=1$

\subsubsection{Special cases T2 - T6}

In the following, we present the equations of special cases of a triaxial ellipsoid, which correspond to the forms T2 T6 of Table 3.

$\mathrm{T} 2:\left[\begin{array}{lll}x & y & z\end{array}\right]\left[\begin{array}{ccc}q_{x x} & q_{x y} & q_{x z} \\ q_{x y} & q_{y y} & q_{y z} \\ q_{x z} & q_{y z} & q_{z z}\end{array}\right]^{-1}\left[\begin{array}{l}x \\ y \\ z\end{array}\right]=1$

$\mathrm{T} 3:\left[\begin{array}{lll}x-t_{x} & y-t_{y} & z-t_{z}\end{array}\right]\left[\begin{array}{ccc}q_{x x} & 0 & 0 \\ 0 & q_{y y} & 0 \\ 0 & 0 & q_{z z}\end{array}\right]^{-1}$

$\left[\begin{array}{l}x-t_{x} \\ y-t_{y} \\ z-t_{z}\end{array}\right]=1$

$\mathrm{T} 4:\left[\begin{array}{lll}x & y & z\end{array}\right]\left[\begin{array}{ccc}q_{x x} & 0 & 0 \\ 0 & q_{y y} & 0 \\ 0 & 0 & q_{z z}\end{array}\right]^{-1}\left[\begin{array}{l}x \\ y \\ z\end{array}\right]=1$ 
Table 2. Parameters for different cases of a triaxial ellipsoid and degenerate cases

\begin{tabular}{|c|c|c|c|c|c|c|c|c|c|}
\hline \multirow[t]{2}{*}{ Case } & \multicolumn{9}{|c|}{ Parameters } \\
\hline & $t_{x}$ & $t_{y}$ & $t_{z}$ & $\boldsymbol{\theta}_{\boldsymbol{x}}$ & $\boldsymbol{\theta}_{y}$ & $\theta_{z}$ & $a_{x}$ & $a_{y}$ & $\boldsymbol{b}$ \\
\hline T1 & $\mathbf{x}$ & $\mathbf{x}$ & $\mathbf{x}$ & $\mathbf{x}$ & $\mathbf{x}$ & $\mathbf{x}$ & $\mathbf{x}$ & $\mathbf{x}$ & $\mathbf{x}$ \\
\hline T2 & 0 & 0 & 0 & $\mathbf{x}$ & $\mathbf{x}$ & $\mathbf{x}$ & $\mathbf{x}$ & $\mathbf{x}$ & $\mathbf{x}$ \\
\hline T3 & $\mathbf{x}$ & $\mathbf{x}$ & $\mathbf{x}$ & 0 & 0 & 0 & $\mathbf{x}$ & $\mathbf{x}$ & $\mathbf{x}$ \\
\hline T4 & 0 & 0 & 0 & 0 & 0 & 0 & $\mathbf{x}$ & $\mathbf{x}$ & $\mathbf{x}$ \\
\hline T5 & $\mathbf{x}$ & $\mathbf{x}$ & $\mathbf{x}$ & 0 & 0 & $\mathbf{x}$ & $\mathbf{x}$ & $x$ & $\mathbf{x}$ \\
\hline T6 & 0 & 0 & 0 & 0 & 0 & $\mathbf{x}$ & $\mathbf{x}$ & $x$ & $\mathbf{x}$ \\
\hline B3 & $\mathbf{x}$ & $\mathbf{x}$ & $\mathbf{x}$ & 0 & 0 & - & \multicolumn{2}{|c|}{$\mathbf{x}$} & $\mathbf{x}$ \\
\hline B4 & 0 & 0 & 0 & 0 & 0 & - & \multicolumn{2}{|c|}{$\mathbf{x}$} & $\mathbf{x}$ \\
\hline S3 & $\mathbf{x}$ & $\mathbf{x}$ & $\mathbf{x}$ & - & - & - & \multicolumn{3}{|c|}{$\mathbf{x}$} \\
\hline S4 & 0 & 0 & 0 & - & - & - & \multicolumn{3}{|c|}{$\mathbf{x}$} \\
\hline
\end{tabular}

$\mathrm{T} 5:\left[\begin{array}{lll}x-t_{x} & y-t_{y} & z-t_{z}\end{array}\right]\left[\begin{array}{ccc}q_{x x} & q_{x y} & 0 \\ q_{x y} & q_{y y} & 0 \\ 0 & 0 & q_{z z}\end{array}\right]^{-1}$

$\left[\begin{array}{l}x-t_{x} \\ y-t_{y} \\ z-t_{z}\end{array}\right]=1$

T6 : $\left[\begin{array}{lll}x & y & z\end{array}\right]\left[\begin{array}{ccc}q_{x x} & q_{x y} & 0 \\ q_{x y} & q_{y y} & 0 \\ 0 & 0 & q_{z z}\end{array}\right]^{-1}\left[\begin{array}{l}x \\ y \\ z\end{array}\right]=1$

\subsection{Oblate spheroid without rotations}

We now present the equations of special cases of an oblate spheroid without rotations, which correspond to the forms B3 and B4 of Table 3.

$$
\begin{aligned}
& \text { B3 : }\left[\begin{array}{ccc}
x-t_{x} & y-t_{y} & z-t_{z}
\end{array}\right]\left[\begin{array}{ccc}
q & 0 & 0 \\
0 & q & 0 \\
0 & 0 & q_{z z}
\end{array}\right]^{-1} \\
& {\left[\begin{array}{l}
x-t_{x} \\
y-t_{y} \\
z-t_{z}
\end{array}\right]=1}
\end{aligned}
$$$$
\text { B4 : }\left[\begin{array}{lll}
x & y & z
\end{array}\right]\left[\begin{array}{ccc}
q & 0 & 0 \\
0 & q & 0 \\
0 & 0 & q_{z z}
\end{array}\right]^{-1}\left[\begin{array}{l}
x \\
y \\
z
\end{array}\right]=1
$$

\subsection{Sphere}

In the degenerate case of a sphere, Eq. (1) is written as

$$
\begin{aligned}
& \mathrm{S} 3:\left[\begin{array}{lll}
x-t_{x} & y-t_{y} & z-t_{z}
\end{array}\right]\left[\begin{array}{lll}
q & 0 & 0 \\
0 & q & 0 \\
0 & 0 & q
\end{array}\right]^{-1} \\
& {\left[\begin{array}{l}
x-t_{x} \\
y-t_{y} \\
z-t_{z}
\end{array}\right]=1}
\end{aligned}
$$

which corresponds to the form S3 of Table 3. Finally, in the special case of a sphere without translation, Eq. (1) is written as

$$
\text { S4: }\left[\begin{array}{lll}
x & y & z
\end{array}\right]\left[\begin{array}{lll}
q & 0 & 0 \\
0 & q & 0 \\
0 & 0 & q
\end{array}\right]^{-1}\left[\begin{array}{l}
x \\
y \\
z
\end{array}\right]=1
$$

which corresponds to the form S4 of Table 3.

The determination of $m_{0}$ elements of vector $\mathbf{c}$ (i.e. of the unknowns) may be accomplished by either an algebraic or a geometric fit to a given set of $k$ points, where $k>m_{0}$, as presented in Sections 3 and 5, respectively.

\subsection{Transformation of vector $\mathrm{c}$ into vector $\mathrm{t}$ and matrix $Q$ (or into parameters)}

In this subsection, we present the transformation of the elements of the vector $\mathbf{c}$ into the vector $\mathbf{t}$ and the matrix $\mathbf{Q}$ for the general case of a triaxial ellipsoid (Case T1). From Eqs. (5) to (7), we have

$$
\mathbf{t}=\left[\begin{array}{c}
t_{x} \\
t_{y} \\
t_{z}
\end{array}\right]=-\left[\begin{array}{ccc}
2 c_{x x} & c_{x y} & c_{x z} \\
c_{x y} & 2 c_{y y} & c_{y z} \\
c_{x z} & c_{y z} & 2 c_{z z}
\end{array}\right]^{-1}\left[\begin{array}{l}
c_{x} \\
c_{y} \\
c_{z}
\end{array}\right]
$$


Table 3. Mathematical models for different cases of a triaxial ellipsoid and degenerate cases

\begin{tabular}{ccc}
\hline Case & $m_{0}$ & Mathematical model \\
\hline T1 & 9 & $c_{x x} x^{2}+c_{y y} y^{2}+c_{z z} z^{2}+c_{x y} x y+c_{x z} x z+c_{y z} y z+c_{x} x+c_{y} y+c_{z} z=1$ \\
\hline T2 & 6 & $c_{x x} x^{2}+c_{y y} y^{2}+c_{z z} z^{2}+c_{x y} x y+c_{x z} x z+c_{y z} y z=1$ \\
\hline T3 & 6 & $c_{x x} x^{2}+c_{y y} y^{2}+c_{z z} z^{2}+c_{x} x+c_{y} y+c_{z} z=1$ \\
\hline T4 & 3 & $c_{x x} x^{2}+c_{y y} y^{2}+c_{z z} z^{2}=1$ \\
\hline T5 & 7 & $c_{x x} x^{2}+c_{y y} y^{2}+c_{z z} z^{2}+c_{x y} x y+c_{x} x+c_{y} y+c_{z} z=1$ \\
\hline T6 & 4 & $c_{x x} x^{2}+c_{y y} y^{2}+c_{z z} z^{2}+c_{x y} x y=1$ \\
\hline B3 & 5 & $c\left(x^{2}+y^{2}\right)+c_{z z} z^{2}+c_{x} x+c_{y} y+c_{z} z=1$ \\
\hline B4 & 2 & $c\left(x^{2}+y^{2}\right)+c_{z z} z^{2}=1$ \\
\hline S3 & 4 & $c\left(x^{2}+y^{2}+z^{2}\right)+c_{x} x+c_{y} y+c_{z} z=1$ \\
\hline S4 & 1 & $c\left(x^{2}+y^{2}+z^{2}\right)=1$ \\
\hline
\end{tabular}

and

$$
\mathbf{Q}=D\left[\begin{array}{ccc}
c_{x x} & \frac{c_{x y}}{2} & \frac{c_{x z}}{2} \\
\frac{c_{x y}}{2} & c_{y y} & \frac{c_{y z}}{2} \\
\frac{c_{x z}}{2} & \frac{c_{y z}}{2} & c_{z z}
\end{array}\right]^{-1}
$$

where

$$
D=1+c_{x x} t_{x}^{2}+c_{y y} t_{y}^{2}+c_{z z} t_{z}^{2}+c_{x y} t_{x} t_{y}+c_{x z} t_{x} t_{z}+c_{y z} t_{y} t_{z}
$$

Once the matrix $\mathbf{Q}$ is determined, we apply the Singular Value Decomposition (SVD is a well-known procedure, included in most mathematical software packages) and we obtain

$$
\mathbf{Q}=\mathbf{R}^{\mathrm{T}} \boldsymbol{\Lambda} \mathbf{R}
$$

which gives directly the orthogonal rotation matrix

$$
\begin{aligned}
\mathbf{R} & =\left[\begin{array}{lll}
r_{11} & r_{12} & r_{13} \\
r_{21} & r_{22} & r_{23} \\
r_{31} & r_{32} & r_{33}
\end{array}\right] \\
& =\left[\begin{array}{ccc}
c_{y} c_{z} & c_{x} s_{z}+s_{x} s_{y} c_{z} & s_{x} s_{z}-c_{x} s_{y} c_{z} \\
-c_{y} s_{z} & c_{x} c_{z}-s_{x} s_{y} s_{z} & s_{x} c_{z}+c_{x} s_{y} s_{z} \\
s_{y} & -s_{x} c_{y} & c_{x} c_{y}
\end{array}\right]
\end{aligned}
$$

where, for instance, $c_{x} s_{z}=\cos \theta_{x} \sin \theta_{z}$, i.e. the rotation angles

$$
\begin{aligned}
& \theta_{x}=\operatorname{atan}\left(\frac{-r_{32}}{r_{33}}\right), \theta_{y}=\operatorname{atan}\left(\frac{r_{31}}{\sqrt{r_{11}^{2}+r_{21}^{2}}}\right), \\
& \theta_{z}=\operatorname{atan}\left(\frac{-r_{21}}{r_{11}}\right)
\end{aligned}
$$

and the diagonal matrix

$$
\boldsymbol{\Lambda}=\left[\begin{array}{ccc}
\lambda_{1} & 0 & 0 \\
0 & \lambda_{2} & 0 \\
0 & 0 & \lambda_{3}
\end{array}\right]
$$

i.e. the semi-axes of the ellipsoid

$$
a_{x}=\sqrt{\lambda_{1}}, \quad a_{y}=\sqrt{\lambda_{2}}, \quad b=\sqrt{\lambda_{3}}
$$

We note that the rows of matrix $\mathbf{R}$ are the components of the unit vectors that correspond to the semi-axes of the ellipsoid. Also, from the $2 \times 2$ sub-matrices of matrix $\mathbf{Q}$ we obtain ellipses on the three planes containing the axes of the $x y z$ system. This facilitates the investigation of the proper sign of the rotation angles. Obviously, we apply the appropriate parts of the transformation in all special and degenerate cases.

\section{Algebraic (linear) fitting}

In this section, we present the process of the algebraic fitting in the general case of a triaxial ellipsoid (T1), in order to obtain the best estimates of elements of vector $\mathbf{c}$. We use the method of least squares, assuming that the Cartesian coordinates of the given points $\left(X_{i}, Y_{i}, Z_{i}\right)$ are of equal precision, uncorrelated and are identical to the coordinates of foot points $\left(x_{i}, y_{i}, z_{i}\right)$. Thus, we can write the linear Eq. (7) in the form

$c_{x x} x_{i}^{2}+c_{y y} y_{i}^{2}+c_{z z} z_{i}^{2}+c_{x y} x_{i} y_{i}+c_{x z} x_{i} z_{i}+c_{y z} y_{i} z_{i}+c_{x} x_{i}+c_{y} y_{i}$
$+c_{z} z_{i}=1+v_{i}, \quad i=1, \ldots, k$

which can be represented in matrix form as

$$
\mathbf{D c}=\mathbf{i}+\boldsymbol{v}
$$

where

$$
\mathbf{D}=\left[\begin{array}{cccc}
x_{1}^{2} & y_{1}^{2} & \cdots & z_{1} \\
\vdots & \vdots & \ddots & \vdots \\
x_{k}^{2} & y_{k}^{2} & \cdots & z_{k}
\end{array}\right]
$$


is a $k \times m_{0}$ matrix containing the coefficients of the elements of vector $\mathbf{c}$ (design matrix), $\mathbf{i}$ is a $k \times 1$ vector with all elements equal to 1 and $\boldsymbol{v}$ is a $k \times 1$ vector of residuals. Then, the solution vector is given by

$$
\mathbf{c}=\left(\mathbf{D}^{\mathrm{T}} \mathbf{D}\right)^{-1} \mathbf{D}^{\mathrm{T}} \mathbf{i}
$$

Subsequently, we can compute the vector $\mathbf{t}$, the rotation matrix $\mathbf{R}$, i.e. the rotation angles and the matrix $\boldsymbol{\Lambda}$, i.e. the semi-axes of the ellipsoid, following the descriptions in subsection 2.4. All special and degenerate cases can be easily obtained by suitable modifications of the matrix $\mathbf{D}$.

\section{Determination of the foot points and statistical indexes}

Before applying the geometric (non-linear) fitting, at first we compute the projections $\left(x_{i}, y_{i}, z_{i}\right)$ of the given points $\left(X_{i}, Y_{i}, Z_{i}\right)$ on the surface of the ellipsoid with the estimated parameters, in the same reference system $x y z$. We first convert the coordinates of the given points $\left(X_{i}, Y_{i}, Z_{i}\right)$ to the reference system $u v w$, aligned to the axes of the estimated ellipsoid, using the known translation vector $\mathbf{t}$ (Eq. (2)) and the rotation matrix R (Eq. (21)), as follows:

$$
\left[\begin{array}{c}
U_{i} \\
V_{i} \\
W_{i}
\end{array}\right]=\left[\begin{array}{lll}
r_{11} & r_{12} & r_{13} \\
r_{21} & r_{22} & r_{23} \\
r_{31} & r_{32} & r_{33}
\end{array}\right]\left[\begin{array}{c}
X_{i}-t_{x} \\
Y_{i}-t_{y} \\
Z_{i}-t_{z}
\end{array}\right], i=1, \ldots, k
$$

For every point $\left(U_{i}, V_{i}, W_{i}\right)$, the desired projection $\left(u_{i}, v_{i}, w_{i}\right)$ can be computed by any of the relevant methods of conversion of Cartesian to geodetic coordinates (e.g. Ligas (2012), Panou and Korakitis (2019)), using the known semi-axes $a_{x}, a_{y}$ and $b$. Finally, the foot points $\left(u_{i}, v_{i}, w_{i}\right)$ are expressed in the reference system $x y z$, as follows:

$$
\begin{aligned}
& {\left[\begin{array}{l}
x_{i} \\
y_{i} \\
z_{i}
\end{array}\right]=\left[\begin{array}{l}
t_{x} \\
t_{y} \\
t_{z}
\end{array}\right]+\left[\begin{array}{lll}
r_{11} & r_{21} & r_{31} \\
r_{12} & r_{22} & r_{32} \\
r_{13} & r_{23} & r_{33}
\end{array}\right]\left[\begin{array}{c}
u_{i} \\
v_{i} \\
w_{i}
\end{array}\right],} \\
& i=1, \ldots, k
\end{aligned}
$$

since $\mathbf{R}^{-1}=\mathbf{R}^{\mathrm{T}}$. From the foot points, we can compute the geoid heights by

$$
\begin{aligned}
N_{i} & =\sqrt{\left(U_{i}-u_{i}\right)^{2}+\left(V_{i}-v_{i}\right)^{2}+\left(W_{i}-w_{i}\right)^{2}} \\
& =\sqrt{\left(X_{i}-x_{i}\right)^{2}+\left(Y_{i}-y_{i}\right)^{2}+\left(Z_{i}-z_{i}\right)^{2}}, \quad i=1, \ldots, k
\end{aligned}
$$

Also, we can compute the statistical indexes $\min (N)$, $\max (N)$,

$$
\operatorname{mean}(N)=\frac{1}{k} \sum_{i=1}^{k} N_{i}
$$

and the root mean square (rms)

$$
r m s(N)=\sqrt{\frac{1}{k} \sum_{i=1}^{k} N_{i}^{2}}
$$

\section{Geometric (non-linear) fitting}

In a similar manner, in this section we present the procedure of the geometric fitting in the general case of a triaxial ellipsoid (T1). For the given points $\left(X_{i}, Y_{i}, Z_{i}\right)$ (assuming unweighted and uncorrelated data) and their foot points $\left(x_{i}, y_{i}, z_{i}\right), i=1, \ldots, k$, the 'observation' equations are

$$
\begin{aligned}
& x_{i}=X_{i}+v_{\chi_{i}} \\
& y_{i}=Y_{i}+v_{Y_{i}} \\
& z_{i}=Z_{i}+v_{Z_{i}}
\end{aligned}
$$

while the constraints are

$g_{i} \dot{=} c_{x x} x_{i}^{2}+c_{y y} y_{i}^{2}+c_{z z} z_{i}^{2}+c_{x y} x_{i} y_{i}+c_{x z} x_{i} z_{i}+c_{y z} y_{i} z_{i}+c_{x} x_{i}$

$+c_{y} y_{i}+c_{z} z_{i}=1$

In the above formulation, we have $n=3 k$ measurements (observations), for the determination of $3 k+$ $m_{0}$ unknowns ( $3 k$ for $\left(x_{i}, y_{i}, z_{i}\right)$ and $m_{0}$ for c) subject to $k$ constraints. Thus, the actual number of unknowns is $m=\left(3 k+m_{0}\right)-k=2 k+m_{0}$. Since the constraints are non-linear equations, we need to linearize all equations using approximate values $\mathbf{r}^{0}=$ $\left[\begin{array}{lllllll}x_{1}^{0} & y_{1}^{0} & z_{1}^{0} & \cdots & x_{k}^{0} & y_{k}^{0} & z_{k}^{0}\end{array}\right]^{T}$ and $\mathbf{c}^{0}$ for the unknowns $\hat{\mathbf{r}}=\mathbf{r}^{0}+\boldsymbol{\delta} \mathbf{r}$ and $\hat{\mathbf{c}}=\mathbf{c}^{0}+\boldsymbol{\delta} \mathbf{c}$. The approximate values $\left(x_{i}^{0}, y_{i}^{0}, z_{i}^{0}\right)$ may be computed as foot points on the ellipsoid with parameters $\mathbf{c}^{0}$ resulting from the algebraic fitting (Section 3), using the approach proposed in Section 4. Thus, the linear approximation of Eqs. (34) to (36) and (37) can be represented in matrix form as

$$
\boldsymbol{\delta} \mathbf{r}=\boldsymbol{\delta} \mathbf{1}+\boldsymbol{v}
$$

and

$$
C \delta \mathbf{r}+\mathbf{D} \delta c=w
$$

where 


$$
\mathbf{C}=\mathbf{J}_{\mathbf{g r}}^{0}=\left[\begin{array}{cccccccccc}
\left.\frac{\partial g_{1}}{\partial x_{1}}\right|^{0} & \left.\frac{\partial g_{1}}{\partial y_{1}}\right|^{0} & \left.\frac{\partial g_{1}}{\partial z_{1}}\right|^{0} & 0 & 0 & 0 & \cdots & 0 & 0 & 0 \\
0 & 0 & 0 & \left.\frac{\partial g_{2}}{\partial x_{2}}\right|^{0} & \left.\frac{\partial g_{2}}{\partial y_{2}}\right|^{0} & \left.\frac{\partial g_{2}}{\partial z_{2}}\right|^{0} & \cdots & 0 & 0 & 0 \\
\vdots & \vdots & \vdots & \vdots & \vdots & \vdots & \ddots & \vdots & \vdots & \vdots \\
0 & 0 & 0 & 0 & 0 & 0 & \cdots & \left.\frac{\partial g_{k}}{\partial x_{k}}\right|^{0} & \left.\frac{\partial g_{k}}{\partial y_{k}}\right|^{0} & \left.\frac{\partial g_{k}}{\partial z_{k}}\right|^{0}
\end{array}\right]
$$

is a $k \times n$ matrix containing the partial derivatives (Jacobian matrix)

$$
\begin{aligned}
& \frac{\partial g_{i}}{\partial x_{i}}=2 c_{x x} x_{i}+c_{x y} y_{i}+c_{x z} z_{i}+c_{x} \\
& \frac{\partial g_{i}}{\partial y_{i}}=2 c_{y y} y_{i}+c_{x y} x_{i}+c_{y z} z_{i}+c_{y} \\
& \frac{\partial g_{i}}{\partial z_{i}}=2 c_{z z} z_{i}+c_{x z} x_{i}+c_{y z} y_{i}+c_{z}
\end{aligned}
$$

$\mathbf{D}=\mathbf{J}_{\mathbf{g c}}^{0}$ is a $k \times m_{0}$ matrix, as in the linear fitting (Eq. (27)) for the approximate values of the foot points $\left(x_{i}^{0}, y_{i}^{0}, z_{i}^{0}\right)$,

$$
\boldsymbol{\delta} \mathbf{1}=\left[\begin{array}{c}
X_{1}-x_{1}^{0} \\
Y_{1}-y_{1}^{0} \\
Z_{1}-z_{1}^{0} \\
\vdots \\
X_{k}-x_{k}^{0} \\
Y_{k}-y_{k}^{0} \\
Z_{k}-z_{k}^{0}
\end{array}\right]
$$

is a $n \times 1$ vector, $\boldsymbol{v}$ is a $n \times 1$ vector of residuals and

$$
\mathbf{w}=\left[\begin{array}{c}
1-g_{1}\left(x_{1}^{0}, y_{1}^{0}, z_{1}^{0}, \mathbf{c}^{0}\right) \\
\vdots \\
1-g_{k}\left(x_{k}^{0}, y_{k}^{0}, z_{k}^{0}, \mathbf{c}^{0}\right)
\end{array}\right]
$$

is a $k \times 1$ vector. The above problem constitutes a least squares problem with constraints which involve additional parameters (see e.g. Mikhail (1976)). Then, the corrections $\boldsymbol{\delta} \mathbf{c}$ and $\boldsymbol{\delta} \mathbf{r}$ to the approximate values, in our case where $\mathbf{A}=\mathbf{I}$ and the weight matrix $\mathbf{P}=\mathbf{I}$, are

$$
\boldsymbol{\delta} \mathbf{c}=\mathbf{L}^{-1} \mathbf{D}^{\mathrm{T}} \mathbf{K}^{-1}(\mathbf{w}-\mathbf{C} \boldsymbol{\delta} \mathbf{l})
$$

and

$$
\boldsymbol{\delta} \mathbf{r}=\boldsymbol{\delta} \mathbf{l}+\mathbf{C}^{\mathrm{T}} \mathbf{K}^{-1}(\mathbf{w}-\mathbf{C} \boldsymbol{\delta} \mathbf{l}-\mathbf{D} \boldsymbol{\delta} \mathbf{c})
$$

where

$$
\mathbf{K}=\mathbf{C C}^{\mathrm{T}}
$$

is a diagonal matrix and

$$
\mathbf{L}=\mathbf{D}^{\mathrm{T}} \mathbf{K}^{-1} \mathbf{D}
$$

Furthermore, the a-posteriori variance factor $\hat{\sigma}_{0}^{2}$ is computed by

$$
\hat{\sigma}_{0}^{2}=\frac{\boldsymbol{v}^{\mathrm{T}} \boldsymbol{v}}{n-m}=\frac{(\boldsymbol{\delta} \mathbf{r}-\boldsymbol{\delta} \mathbf{1})^{\mathrm{T}}(\boldsymbol{\delta} \mathbf{r}-\boldsymbol{\delta} \mathbf{l})}{k-m_{0}}
$$

where $n-m$ represents the degrees of freedom, while the vector of residuals $\boldsymbol{v}$ is computed from Eq. (38). Finally, the a-posteriori variance-covariance matrix of the vector $\hat{\mathbf{c}}$ is given by

$$
\hat{\mathbf{V}}_{\hat{\mathbf{c}}}=\hat{\sigma}_{0}^{2} \mathbf{L}^{-1}
$$


We then compute the coordinates of the ellipsoid center, the rotation angles and the semi-axes of the ellipsoid, according to the description in subsection 2.4 .

Theoretically, the solution process is iterative until the corrections to the approximate values become negligible. It is worth emphasizing that the minimization problem solved in the geometric approach satisfies the equation $\sum_{i=1}^{k} N_{i}^{2}=\min$. This can be checked by repeating the procedure described in Section 4 and computing new statistic indexes $\min (\hat{N}), \max (\hat{N})$, mean $(\hat{N})$ and $r m s(\hat{N})$.

\subsection{Uncertainty estimates}

Assuming that $c_{x y} \cong c_{x z} \cong c_{y z} \approx 0$ and using Eq. (17), the coordinates of the ellipsoid's center are computed by

$$
\begin{aligned}
t_{x} & =-\frac{1}{2} \frac{c_{x}}{c_{x x}} \\
t_{y} & =-\frac{1}{2} \frac{c_{y}}{c_{y y}} \\
t_{z} & =-\frac{1}{2} \frac{c_{z}}{c_{z z}}
\end{aligned}
$$

Thus, their uncertainties $\sigma$ may be computed by applying the error propagation law

$$
\begin{aligned}
\sigma_{t_{x}} & = \pm \frac{1}{2} \sqrt{\left(\frac{c_{x}}{c_{x x}^{2}}\right)^{2} \sigma_{c_{x x}}^{2}+\left(-\frac{1}{c_{x x}}\right)^{2} \sigma_{c_{x}}^{2}} \\
\sigma_{t_{y}} & = \pm \frac{1}{2} \sqrt{\left(\frac{c_{y}}{c_{y y}^{2}}\right)^{2} \sigma_{c_{y y}}^{2}+\left(-\frac{1}{c_{y y}}\right)^{2} \sigma_{c_{y}}^{2}} \\
\sigma_{t_{z}} & = \pm \frac{1}{2} \sqrt{\left(\frac{c_{z}}{c_{z z}^{2}}\right)^{2} \sigma_{c_{z z}}^{2}+\left(-\frac{1}{c_{z z}}\right)^{2} \sigma_{c_{z}}^{2}}
\end{aligned}
$$

where $\sigma_{c}$ are the uncertainties of the elements of vector $\mathbf{c}$, as computed from the fitting. We note that the uncertainty of the ellipsoid semi-axes can be approximated by

$$
\sigma_{a_{x}} \cong \sigma_{a_{y}} \cong \sigma_{b} \approx \frac{\hat{\sigma}_{0}}{\sqrt{k}}
$$

where $k$ is the number of points of fitting. Finally, the uncertainty of the orientation of the ellipsoid, can be approximated by

$$
\sigma_{\theta_{x}} \cong \sigma_{\theta_{y}} \approx \frac{\hat{\sigma}_{0}}{b \sqrt{k}}
$$

and

$$
\sigma_{\theta_{z}} \approx \frac{\hat{\sigma}_{0}}{a_{y} \sqrt{k}}
$$

\section{Data and numerical results}

\subsection{Data}

The methodology described above was applied to an extensive set of points in space, corresponding to points of the geoid, at particular values of geodetic coordinates $\varphi$ and $\lambda$, using values of the geoid undulation $N$ provided by the ICGEM service for several gravity field models.

The grid of points used was roughly equidistant, with a spatial resolution determined by the maximum degree of the gravity field model. For example, the model GOCO06s has a maximum degree 300 , so the equivalent resolution (0.6 degrees) corresponds to a spatial resolution (half wavelength) of about $67 \mathrm{~km}$ at the equator.

The geodetic coordinates of the grid were determined by setting up a number of points along circles of equal latitude, separated by the given resolution. The angular separation along these circles (longitude difference) was increased at each latitude step (kept constant at the value of the angular resolution of the model) so that the spatial distance of points along each latitude circle remained roughly constant. In this way, the resulting grid has a uniform surface density of points, in contrast to a regular grid of $(\varphi, \lambda)$ values, which is denser towards the poles (dataset D1.1 in Table 4). The geodetic coordinates $\left(\varphi_{i}, \lambda_{i}, N_{i}\right)$ of a point on the geoid are related to the corresponding Cartesian coordinates $\left(X_{i}, Y_{i}, Z_{i}\right)$ by well-known expressions, where $h_{i} \equiv N_{i}$. In the case of WGS 84 we have: major semiaxis $a=6378137.0 \mathrm{~m}$ and flattening $f=\frac{1}{298.257223563}$ (minor semiaxis $b=a(1-f)$ ) (NIMA (2000)).

In the ellipsoid fitting we used six different gravity field models, which represent different stages of our knowledge about the gravity field in the last fifty years, as follows:

- GOCO06s, described by Kvas et al. (2019)

- EGM2008, described by Pavlis et al. (2012)

- EGM96, described by Lemoine et al. (1998)

- OSU86f, described by Rapp and Cruz (1986)

- GEM8, described by Wagner et al. (1977)

- SE1, described by Lundquist and Veis (1966)

For more details regarding the evaluation of these models, please visit http://icgem.gfz-potsdam.de/home.html.

Table 4 summarizes the relevant data for each data set, as well as some important statistical properties of each model for the particular grid of points used.

We should note that the great values of the mean and rms quantities for the SE1 model are due to the fact that the oblate spheroid used at the time was different from 
Table 4. Characteristics of the data sets used

\begin{tabular}{|c|c|c|c|c|c|c|c|}
\hline \multirow{2}{*}{ Dataset } & \multirow{2}{*}{ Geoid model } & \multirow{2}{*}{ Spatial resolution $(\mathrm{km})$} & \multirow{2}{*}{ Number of points } & \multicolumn{4}{|c|}{ Statistics } \\
\hline & & & & $\begin{array}{c}\operatorname{mean}(N) \\
(\mathrm{m})\end{array}$ & $\min (N)(\mathrm{m})$ & $\begin{array}{c}\max (N) \\
(\mathrm{m})\end{array}$ & $r m s(N)(m)$ \\
\hline D1.1 & \multirow{2}{*}{$\begin{array}{l}\text { G0C006s } \\
( \pm 0.15 \mathrm{~m})\end{array}$} & Variable & 179402 & -0.90 & -106.27 & 84.93 & 29.28 \\
\hline D1.2 & & 67 & 114446 & -0.05 & -106.33 & 85.31 & 30.59 \\
\hline D2.1 & \multirow{4}{*}{$\begin{array}{r}\text { EGM2008 } \\
( \pm 0.15 \mathrm{~m})\end{array}$} & 56 & 164836 & -0.06 & -106.43 & 85.92 & 30.59 \\
\hline D2.2 & & 67 & 114446 & -0.06 & -106.26 & 85.31 & 30.60 \\
\hline D2.3 & & 111 & 41164 & -0.06 & -105.93 & 85.92 & 30.60 \\
\hline D2.4 & & 1334 & 286 & -0.13 & -94.59 & 72.39 & 30.59 \\
\hline D3 & $\begin{array}{l}\text { EGM96 } \\
( \pm 0.50 \mathrm{~m})\end{array}$ & 56 & 164836 & -0.05 & -106.48 & 85.44 & 30.59 \\
\hline D4 & $\begin{array}{l}\text { OSU86f } \\
( \pm 1.5 \mathrm{~m}) \\
\end{array}$ & 56 & 164836 & 0.02 & -107.21 & 82.20 & 30.53 \\
\hline D5 & $\begin{array}{r}\text { GEM8 } \\
( \pm 3 \mathrm{~m}) \\
\end{array}$ & 801 & 769 & 0.13 & -102.14 & 74.42 & 30.58 \\
\hline D6 & SE1 $( \pm 10 m)$ & 1334 & 286 & 44.12 & -34.74 & 101.20 & 51.83 \\
\hline
\end{tabular}

the WGS 84. In any case, the particulars of the reference spheroid do not affect the preparation of the data sets. Therefore, we decided to use WGS 84 as a common reference for all models, since it is also more realistic than the older reference surfaces.

In all numerical computations that are presented in the following sections, we used a personal computer running a 64-bit Linux Debian operating system, with an Intel Core i5-2430M CPU (clocked at $2.4 \mathrm{GHz}$ ) and 6 GB RAM. All algorithms were coded in $\mathrm{C}++$ and compiled by the opensource GNU GCC compiler (Level 2 optimization). The computations for the fittings (both algebraic and geometric) were performed at quad precision ( 33 digits accuracy) using the open-source "libquadmath", the GCC Quad Precision Math Library. The computations for the foot points, following the method presented in Panou and Korakitis (2019), were performed using the "long double" data type, which provides an accuracy of 18 digits.

\subsection{Results and statistics for the geoid model G0C006s}

Using the data sets derived from the GOCO06s geoid model (a satellite-only model) we obtained the results for the ellipsoid parameters and the relevant statistics for all cases as presented in Tables 5 and 6, respectively.

Looking at the differences in the parameter values from the algebraic fitting in the $\mathrm{T} 1$ case between the data sets D1.1 and D1.2, one realizes the importance of using a data set of points with uniform surface density (almost equally spaced points). We also note some minor differences in the values for the rotation angles, which justifies our decision to use the geometric fitting for the study of all subsequent cases and geoid models.

From the results presented in Table 6 it is apparent that the translation parameters $t_{x}, t_{y}$ and $t_{z}$, as well as the rotation angles $\theta_{x}$ and $\theta_{y}$, have a very small impact on the statistics of the fitting. Therefore, we added the row ' $\mathrm{T} 6$ ' in both Tables 5 and 6 , which represent rounded values of the parameters. We also note the agreement of the statistics of the WGS 84 initial data and the statistics of fitting an oblate spheroid (case G - B4 above). In contrast, we note the improvement of the statistics when fitting a triaxial ellipsoid (case G - T6 above). This improvement is visualized in the following Figures 1 to 4 . Figures 1 to 3 were created by the free software GMT 6 (Wessel et al. (2019)). Figures 1 and 2 present the same quantity (geoid height) with respect to two different reference surfaces, while Figure 3 depicts the difference between the reference surfaces themselves.

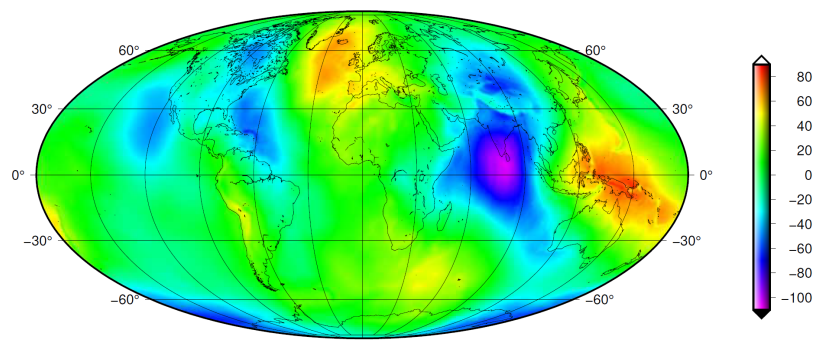

Fig. 1. Geoidal heights (in meters) of model G0C006s with respect to the oblate spheroid WGS 84 in Mollweide projection. mean = $-0.05 \mathrm{~m}, \min =-106.33 \mathrm{~m}, \max =85.31 \mathrm{~m}, r m s=30.59 \mathrm{~m}$. 
Table 5. Resulting values of the parameters of fitting for the geoid model GOCO06s

\begin{tabular}{|c|c|c|c|c|c|c|c|c|c|c|}
\hline \multirow{2}{*}{ Dataset } & \multirow{2}{*}{ Fitting - Case } & \multicolumn{9}{|c|}{ Parameters } \\
\hline & & $\begin{array}{c}t_{x} \\
(\mathrm{~m})\end{array}$ & $\begin{array}{c}t_{y} \\
(\mathrm{~m})\end{array}$ & $\begin{array}{c}t_{z} \\
(\mathrm{~m})\end{array}$ & $\theta_{x}\left({ }^{\circ}\right)$ & $\theta_{y}\left(^{\circ}\right)$ & $\theta_{z}\left(^{\circ}\right)$ & $a_{x}(\mathrm{~m})$ & $a_{y}(\mathrm{~m})$ & $b(m)$ \\
\hline D1.1 & A - T1 & 5.43 & 0.55 & 6.93 & -0.0048 & -0.0056 & -13.9666 & 6378173.07 & 6378102.94 & 6356749.49 \\
\hline \multirow{21}{*}{ D1.2 } & A - T1 & -0.08 & -0.05 & -0.04 & -0.000020 & -0.000056 & -14.9283215 & 6378171.918 & 6378102.064 & 6356752.169 \\
\hline & $G-T 1$ & -0.11 & -0.05 & -0.06 & 0.0000043 & -0.000083 & -14.9366733 & 6378171.915 & 6378102.061 & 6356752.175 \\
\hline & G-II & \pm 0.13 & \pm 0.13 & \pm 0.13 & \pm 0.0000007 & \pm 0.0000007 & \pm 0.0000007 & \pm 0.07 & \pm 0.07 & $\mathbf{\pm 0 . 0 7}$ \\
\hline & G - T2 & 0 & 0 & 0 & 0.0000043 & -0.000083 & -14.9366732 & 6378171.92 & 6378102.06 & 6356752.17 \\
\hline & & & & & \pm 0.0000007 & \pm 0.0000007 & \pm 0.0000007 & \pm 0.07 & \pm 0.07 & \pm 0.07 \\
\hline & G - T3 & -0.11 & -0.05 & -0.06 & 0 & 0 & 0 & 6378167.27 & 6378106.70 & 6356752.17 \\
\hline & $G-13$ & \pm 0.13 & \pm 0.13 & \pm 0.13 & 0 & 0 & 0 & \pm 0.08 & \pm 0.08 & \pm 0.08 \\
\hline & G - T4 & 0 & 0 & 0 & 0 & 0 & 0 & $\begin{array}{c}6378167.27 \\
\pm 0.08\end{array}$ & $\begin{array}{c}6378106.70 \\
\pm 0.08\end{array}$ & $\begin{array}{c}6356752.17 \\
\pm 0.08\end{array}$ \\
\hline & $G-T 5$ & -0.11 & -0.05 & -0.06 & 0 & 0 & -14.9366733 & 6378171.92 & 6378102.06 & 6356752.17 \\
\hline & $0-10$ & \pm 0.13 & \pm 0.13 & \pm 0.13 & 0 & 0 & \pm 0.0000007 & $\mathbf{\pm 0 . 0 7}$ & $\mathbf{\pm 0 . 0 7}$ & \pm 0.07 \\
\hline & G - T6 & 0 & 0 & 0 & 0 & 0 & -14.9366732 & 6378171.92 & 6378102.06 & 6356752.17 \\
\hline & $0-10$ & 0 & 0 & 0 & 0 & 0 & \pm 0.0000007 & \pm 0.07 & \pm 0.07 & $\mathbf{\pm 0 . 0 7}$ \\
\hline & T6 & 0 & 0 & 0 & 0 & 0 & -14.9367 & 6378171.92 & 6378102.06 & 6356752.17 \\
\hline & $G-R_{3}$ & -0.11 & -0.05 & -0.06 & 0 & 0 & - & \multicolumn{2}{|c|}{6378136.99} & 6356752.17 \\
\hline & J & \pm 0.16 & \pm 0.16 & \pm 0.01 & 0 & 0 & 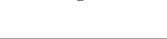 & \multicolumn{2}{|c|}{ \pm 0.09} & \pm 0.09 \\
\hline & $G-B 4$ & 0 & 0 & 0 & 0 & 0 & - & \multicolumn{2}{|c|}{6378136.99} & 6356752.17 \\
\hline & - D4 & 0 & $\mathbf{0}$ & & 0 & 0 & 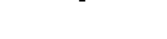 & \multicolumn{2}{|c|}{ \pm 0.09} & \pm 0.09 \\
\hline & $G-53$ & -0.08 & -0.05 & -0.04 & - & - & - & \multirow{2}{*}{\multicolumn{3}{|c|}{$\begin{array}{c}6371037 \\
\pm 19\end{array}$}} \\
\hline & Sב- G & \pm 33 & \pm 33 & \pm 33 & & & & & & \\
\hline & G - S4 & 0 & 0 & 0 & - & - & - & \multicolumn{3}{|c|}{6371037} \\
\hline & G-S4 & 0 & 0 & 0 & 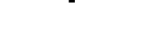 & 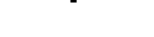 & 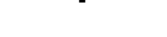 & \multicolumn{3}{|c|}{ \pm 19} \\
\hline
\end{tabular}

Table 6. Statistics of fitting for the geoid model G0C006s

\begin{tabular}{|c|c|c|c|c|c|c|}
\hline \multirow{2}{*}{ Dataset } & \multirow{2}{*}{ Fitting - Case } & \multicolumn{5}{|c|}{ Statistics } \\
\hline & & $a_{x}-a_{y}(\mathrm{~m})$ & mean $(\hat{N})(\mathrm{m})$ & $\min (\hat{N})(\mathrm{m})$ & $\max (\hat{N})(\mathrm{m})$ & $r m s(\hat{\boldsymbol{N}})(\mathrm{m})$ \\
\hline D1.1 & A - T1 & 70.13 & -0.0002 & -74.48 & 71.73 & 24.29 \\
\hline \multirow{12}{*}{ D1.2 } & A - T1 & 69.854 & -0.0002 & -71.90 & 70.30 & 24.70 \\
\hline & G - T1 & 69.854 & 0.000001 & -71.89 & 70.28 & 24.70 \\
\hline & G - T2 & 69.86 & 0.000001 & -71.96 & 70.29 & 24.70 \\
\hline & G - T3 & 60.57 & 0.000001 & -78.36 & 86.29 & 26.29 \\
\hline & G - T4 & 60.57 & 0.000001 & -78.43 & 86.30 & 26.29 \\
\hline & G - T5 & 69.86 & 0.000001 & -71.88 & 70.28 & 24.70 \\
\hline & G - T6 & 69.86 & 0.000001 & -71.96 & 70.29 & 24.70 \\
\hline & T6 & 69.86 & 0.0001 & -71.96 & 70.29 & 24.70 \\
\hline & G - B3 & 0 & 0.000001 & -106.24 & 85.25 & 30.59 \\
\hline & G - B4 & 0 & 0.000001 & -106.32 & 85.33 & 30.59 \\
\hline & G - S3 & 0 & -0.00006 & -14314 & 7171 & 6365 \\
\hline & G - S4 & 0 & -0.00006 & -14314 & 7177 & 6365 \\
\hline
\end{tabular}




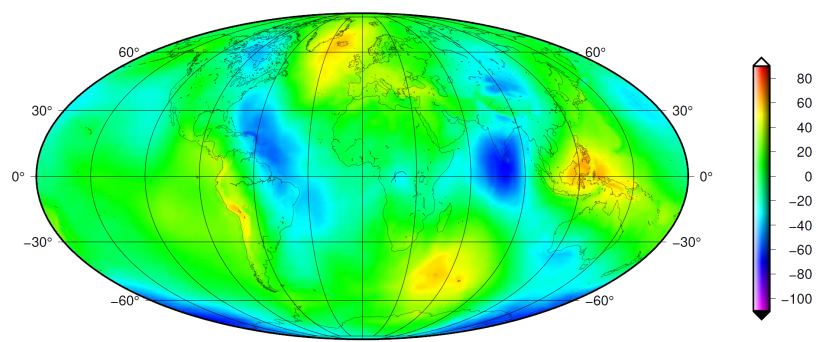

Fig. 2. Geoidal heights (in meters) of model G0C006s with respect to the computed triaxial ellipsoid of case G-T6 in Mollweide projection. mean $=0.000001 \mathrm{~m}, \min =-71.96 \mathrm{~m}, \max =70.29 \mathrm{~m}$, $r m s=24.70 \mathrm{~m}$.

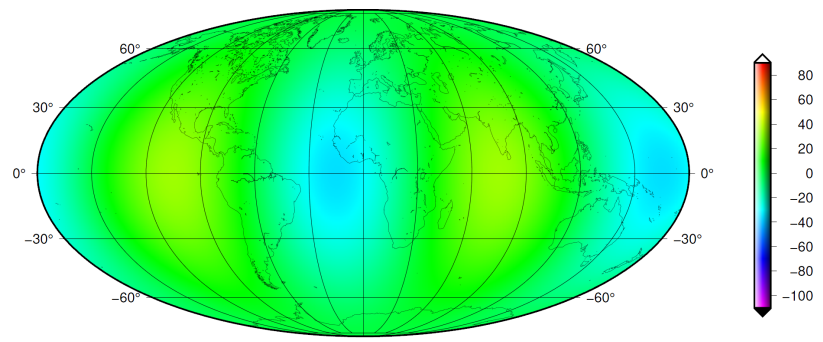

Fig. 3. Geoidal heights given in Fig. 2 minus geoidal heights given in Fig. 1 (in meters) in Mollweide projection. mean $=0.05 \mathrm{~m}, \min =$ $-34.92 \mathrm{~m}, \max =34.94 \mathrm{~m}$.

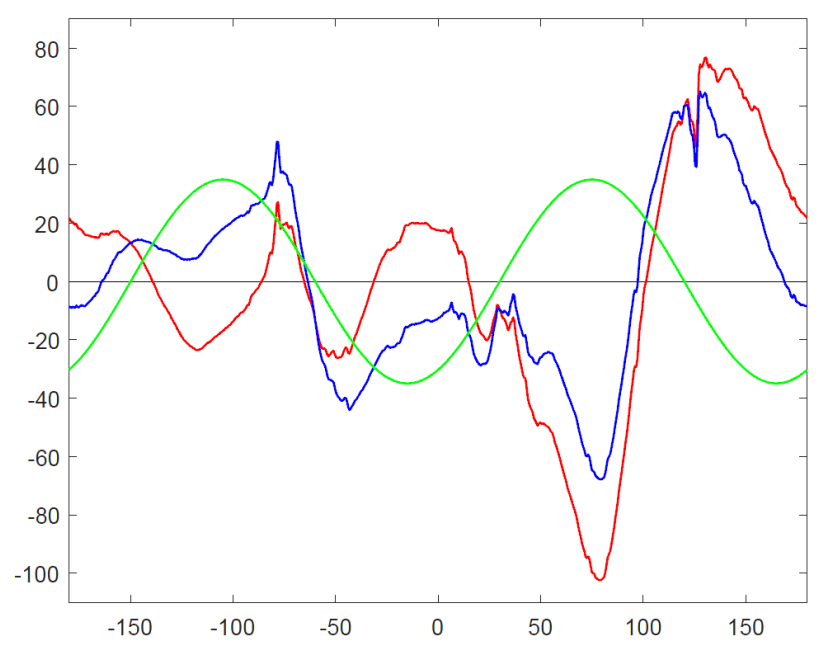

Fig. 4. Geoidal heights (in meters - vertical axis) in a cross section along equator (in degrees - horizontal axis) of model G0C006s with respect to: i) the oblate spheroid WGS 84 (red line) and ii) the computed triaxial ellipsoid of case G-T6 (blue line). The green line illustrates the difference (in meters) of the two heights (heights given in blue line minus heights given in red line).

\subsection{Results and statistics for the geoid model EGM2008}

In order to study the effect of the spatial resolution of the data on the parameters of the fitting, we prepared four dif- ferent data sets, all based on the geoid model EGM2008, at different resolutions, corresponding to the ones of the other geoid models. The results of the geometric fitting and the relevant statistics (cases G - T6 and G - B4) are presented in Table 7.

From the above values we conclude that the effect of the spatial resolution is insignificant, in the sense that the values are in agreement considering their statistical error bounds.

\subsection{Results and statistics for various geoid models}

In order to study the performance of different geoid models, going back roughly 50 years ago, we prepared data sets for four geoid models of the past, which were proposed about every 10 years. The results of the geometric fitting and the relevant statistics (cases G - T6 and G - B4) are presented in Table 8.

From the above values we conclude that the estimates for the difference between the equatorial semi-axes, as well as for the longitude of the semi-major axis $\theta_{z}$, are almost the same.

\subsection{Comparison with results from spherical harmonic coefficients}

For a near-spherical body, using the spherical harmonic coefficients of degree and order two of a global gravity field model, we can compute the longitude of the principal axis of the least moment of inertia by:

$$
\lambda_{0}=\frac{1}{2} \tan ^{-1}\left(\frac{S_{22}}{C_{22}}\right)
$$

and using the reference radius $R$ (which only has the meaning of a scale factor) one gets an expression for the (positive) difference of the two equatorial semi-axes (Liu and Chao (1991))

$$
a_{x}-a_{y}=R \sqrt{15} \sqrt{C_{22}^{2}+S_{22}^{2}}
$$

The uncertainties $\sigma$ of the parameters in Eqs. (61) and (62) can be computed by applying the error propagation law:

$$
\sigma_{\lambda_{0}}= \pm \frac{1}{2\left(C_{22}^{2}+S_{22}^{2}\right)} \sqrt{S_{22}^{2} \sigma_{C_{22}}^{2}+C_{22}^{2} \sigma_{S_{22}}^{2}}
$$

and

$$
\sigma_{a_{x}-a_{y}}= \pm \frac{R \sqrt{15}}{\sqrt{C_{22}^{2}+S_{22}^{2}}} \sqrt{C_{22}^{2} \sigma_{C_{22}}^{2}+S_{22}^{2} \sigma_{S_{22}}^{2}}
$$


Table 7. Resulting values of the parameters and statistics of fitting for the geoid model EGM2008

\begin{tabular}{|c|c|c|c|c|c|c|c|c|c|c|}
\hline \multirow{2}{*}{ Dataset } & \multirow[t]{2}{*}{ Fitting - Case } & \multicolumn{4}{|c|}{ Parameters } & \multicolumn{5}{|c|}{ Statistics } \\
\hline & & $\theta_{z}\left(^{\circ}\right)$ & $a_{x}(m)$ & $a_{y}(\mathrm{~m})$ & $b(\mathrm{~m})$ & $\begin{array}{c}a_{x}-a_{y} \\
(\mathrm{~m})\end{array}$ & $\underset{(\mathrm{m})}{\operatorname{mean}}(\hat{\boldsymbol{N}})$ & $\begin{array}{c}\min (\hat{N}) \\
(\mathrm{m})\end{array}$ & $\underset{(\mathrm{m})}{\max }(\hat{N})$ & $\begin{array}{c}r m s(\hat{N}) \\
(\mathrm{m})\end{array}$ \\
\hline \multirow[t]{2}{*}{ D2.1 } & G - T6 & $\begin{array}{l}-14.9356740 \\
\pm 0.0000005\end{array}$ & $\begin{array}{l}6378171.88 \\
\pm 0.06\end{array}$ & $\begin{array}{l}6378102.03 \\
\pm 0.06\end{array}$ & $\begin{array}{l}6356752.24 \\
\pm 0.06\end{array}$ & 69.85 & 0.000001 & -71.97 & 70.49 & 24.70 \\
\hline & G - B4 & - & \multicolumn{2}{|c|}{$\begin{array}{c}6378136.96 \\
\pm 0.08\end{array}$} & $\begin{array}{l}6356752.24 \\
\pm 0.08\end{array}$ & 0 & 0.00001 & -106.38 & 85.97 & 30.59 \\
\hline \multirow[t]{2}{*}{ D2.2 } & G - T6 & $\begin{array}{l}-14.9369405 \\
\pm 0.0000005\end{array}$ & $\begin{array}{l}6378171.90 \\
\pm 0.07\end{array}$ & $\begin{array}{l}6378102.04 \\
\pm 0.07\end{array}$ & $\begin{array}{l}6356752.20 \\
\pm 0.07\end{array}$ & 69.86 & 0.000001 & -71.87 & 70.45 & 24.71 \\
\hline & G - B4 & - & \multicolumn{2}{|c|}{$\begin{array}{c}6378136.97 \\
\pm 0.09 \\
\end{array}$} & $\begin{array}{l}6356752.20 \\
\pm 0.09\end{array}$ & 0 & 0.000001 & -106.23 & 85.34 & 30.60 \\
\hline \multirow[t]{2}{*}{ D2.3 } & G - T6 & $\begin{array}{c}-14.939350 \\
\pm 0.000001 \\
\end{array}$ & $\begin{array}{l}6378171.88 \\
\pm 0.12\end{array}$ & $\begin{array}{l}6378102.03 \\
\pm 0.12 \\
\end{array}$ & $\begin{array}{l}6356752.24 \\
\pm 0.12 \\
\end{array}$ & 69.85 & 0.000001 & -71.46 & 67.43 & 24.70 \\
\hline & G - B4 & - & \multicolumn{2}{|c|}{$\begin{array}{c}6378136.95 \\
\pm 0.15 \\
\end{array}$} & $\begin{array}{l}6356752.24 \\
\pm 0.15\end{array}$ & 0 & 0.000001 & -105.89 & 85.97 & 30.60 \\
\hline \multirow[t]{2}{*}{ D2.4 } & G - T6 & $\begin{array}{l}-15.12522 \\
\pm 0.00001\end{array}$ & $\begin{array}{l}6378172.0 \\
\pm 1.5\end{array}$ & $\begin{array}{l}6378102.1 \\
\quad \pm 1.5\end{array}$ & $\begin{array}{c}6356751.8 \\
\pm 1.5\end{array}$ & 69.9 & 0.000001 & -64.19 & 60.03 & 24.75 \\
\hline & G - B4 & - & \multicolumn{2}{|c|}{$6378137.1 \pm 1.8$} & $\begin{array}{l}6356751.8 \\
\pm 1.8\end{array}$ & 0 & 0.000001 & -94.65 & 72.33 & 30.59 \\
\hline
\end{tabular}

Table 8. Resulting values of the parameters and statistics of fitting for various geoid models

\begin{tabular}{|c|c|c|c|c|c|c|c|c|c|c|}
\hline \multirow[t]{2}{*}{ Dataset } & \multirow[t]{2}{*}{ Fitting - Case } & \multicolumn{4}{|c|}{ Parameters } & \multicolumn{5}{|c|}{ Statistics } \\
\hline & & $\boldsymbol{\theta}_{\boldsymbol{z}}\left(^{\circ}\right)$ & $a_{x}(\mathrm{~m})$ & $a_{y}(\mathrm{~m})$ & $b(\mathrm{~m})$ & $\begin{array}{c}a_{x}-a_{y} \\
(\mathrm{~m})\end{array}$ & $\underset{(\mathrm{m})}{\operatorname{mean}}(\hat{N})$ & $\underset{(\mathrm{m})}{\min }(\hat{N})$ & $\underset{(\mathrm{m})}{\max }(\hat{N})$ & $\begin{array}{c}r m s \\
\text { (m) }\end{array}$ \\
\hline \multirow[t]{2}{*}{ D3 } & G - T6 & $\begin{array}{l}-14.9366367 \\
\pm 0.0000005\end{array}$ & $\begin{array}{l}6378171.88 \\
\pm 0.06\end{array}$ & $\begin{array}{l}6378102.03 \\
\pm 0.06\end{array}$ & $\begin{array}{c}6356752.23 \\
\pm 0.06\end{array}$ & 69.85 & 0.000001 & -72.03 & 69.85 & 24.70 \\
\hline & G - B4 & - & \multicolumn{2}{|c|}{$\begin{array}{c}6378136.96 \\
\pm 0.08\end{array}$} & $\begin{array}{c}6356752.23 \\
\pm 0.08\end{array}$ & 0 & 0.000001 & -106.44 & 85.48 & 30.59 \\
\hline \multirow[t]{2}{*}{ D4 } & G - T6 & $\begin{array}{l}-14.9328972 \\
\pm 0.0000005\end{array}$ & $\begin{array}{l}6378171.95 \\
\pm 0.06\end{array}$ & $\begin{array}{c}6378102.13 \\
\pm 0.06\end{array}$ & $\begin{array}{l}6356752.30 \\
\pm 0.06\end{array}$ & 69.82 & 0.000001 & -72.90 & 66.13 & 24.63 \\
\hline & G - B4 & - & \multicolumn{2}{|c|}{$\begin{array}{c}6378137.04 \\
\pm 0.08\end{array}$} & $\begin{array}{l}6356752.30 \\
\pm 0.08\end{array}$ & 0 & 0.000001 & -107.25 & 82.16 & 30.53 \\
\hline \multirow[t]{2}{*}{ D5 } & G - T6 & $\begin{array}{l}-14.929005 \\
\pm 0.000008\end{array}$ & $\begin{array}{l}6378171.7 \\
\pm 0.9\end{array}$ & $\begin{array}{l}6378102.0 \\
\pm 0.9\end{array}$ & $\begin{array}{l}6356753.0 \\
\pm 0.9\end{array}$ & 69.7 & 0.000001 & -67.88 & 68.04 & 24.59 \\
\hline & G - B4 & - & \multicolumn{2}{|c|}{$\begin{array}{c}6378136.9 \\
\pm 1.1\end{array}$} & $\begin{array}{l}6356753.0 \\
\pm 1.1\end{array}$ & 0 & 0.000001 & -102.01 & 74.55 & 30.58 \\
\hline \multirow[t]{2}{*}{ D6 } & G - T6 & $\begin{array}{l}-14.82805 \\
\pm 0.00001\end{array}$ & $\begin{array}{c}6378215.3 \\
\pm 1.2\end{array}$ & $\begin{array}{c}6378147.4 \\
\pm 1.2\end{array}$ & $\begin{array}{c}6356796.0 \\
\pm 1.2\end{array}$ & 67.9 & 0.000001 & -66.82 & 58.78 & 20.84 \\
\hline & G - B4 & - & \multicolumn{2}{|c|}{$\begin{array}{c}6378181.4 \\
\pm 1.6\end{array}$} & $\begin{array}{l}6356796.0 \\
\pm 1.6\end{array}$ & 0 & 0.000001 & -79.10 & 57.23 & 27.20 \\
\hline
\end{tabular}


Table 9. Data and estimated values for the parameters for the geoid models

\begin{tabular}{|c|c|c|c|c|c|}
\hline $\begin{array}{l}\text { Geoid } \\
\text { model }\end{array}$ & $R(\mathrm{~m})$ & $C_{22}$ & $S_{22}$ & $\lambda_{0}\left(^{\circ}\right)$ & $a_{x}-a_{y}(\mathrm{~m})$ \\
\hline GoC006s & 6378136.3 & $\begin{array}{c}2.439370388690 \cdot 10^{-6} \\
\pm 8.092959988764 \cdot 10^{-13}\end{array}$ & $\begin{array}{l}-1.400307620664 \cdot 10^{-6} \\
\pm 9.226405195253 \cdot 10^{-13}\end{array}$ & $\begin{array}{c}-14.9288750 \\
\pm 0.0000091\end{array}$ & $\begin{array}{l}69.480959 \\
\pm 0.000021\end{array}$ \\
\hline EGM2008 & 6378136.3 & $\begin{array}{c}2.43938357328313 \cdot 10^{-6} \\
\pm 7.230231722 \cdot 10^{-12} \\
\end{array}$ & $\begin{array}{c}-1.40027370385934 \cdot 10^{-6} \\
\pm 7.425816951 \cdot 10^{-12}\end{array}$ & $\begin{array}{c}-14.928509 \\
\pm 0.000075 \\
\end{array}$ & $\begin{array}{r}69.48082 \\
\pm 0.00018 \\
\end{array}$ \\
\hline EGM96 & 6378136.3 & $\begin{array}{c}2.43914352398 \cdot 10^{-6} \\
\pm 5.3739154 \cdot 10^{-11}\end{array}$ & $\begin{array}{c}-1.40016683654 \cdot 10^{-6} \\
\pm 5.4353269 \cdot 10^{-11}\end{array}$ & $\begin{array}{c}-14.92878 \\
\pm 0.00055\end{array}$ & $\begin{array}{l}69.4744 \\
\pm 0.0013 \\
\end{array}$ \\
\hline OSU86f & 6378136.3 & $2.43834012895 \cdot 10^{-6}$ & $-1.39928194222 \cdot 10^{-6}$ & -14.92504 & 69.4463 \\
\hline GEM8 & 6378136.3 & $2.4345 \cdot 10^{-6}$ & $-1.3953 \cdot 10^{-6}$ & -14.90929 & 69.3151 \\
\hline SE1 & 6378165 & $2.379 \cdot 10^{-6}$ & $-1.351 \cdot 10^{-6}$ & -14.79581 & 67.5823 \\
\hline
\end{tabular}

where $\sigma_{C}$ and $\sigma_{S}$ are the uncertainties of the coefficients, as given in a global gravity field model.

Table 9 presents the data and the estimated values for the above parameters for each one of the geoid models used.

From the estimates in Table 9 we note the high degree of precision of these values, as well as their consistency and the very small difference from the results of the corresponding geometric fitting. Finally, we note that all geoid models do not include the spherical harmonic coefficients of degree one, implying that the translations are assumed zero.

\section{Conclusions and future work}

In this work, we present a procedure for the estimation of parameters of Earth's triaxiality through a geometric fitting of a triaxial ellipsoid to a set of given points in space, derived from and representing various geoid models. The condition for the fitting is the minimization of the sum of the squared geoid heights. Taking into consideration the advantages of a satellite-only model (GOCO06s), we conclude that the optimum semi-axes of the triaxial ellipsoid with four parameters are equal to $6378171.92 \mathrm{~m}, 6378102.06$ $\mathrm{m}$ and $6356752.17 \mathrm{~m}$, while the longitude of the equatorial major semi-axis is -14.9367 degrees. This reference surface leads to significantly reduced values of the geoid heights: the range is reduced from $191.64 \mathrm{~m}$ to $142.25 \mathrm{~m}(25 \%)$ and rms value is reduced from $30.59 \mathrm{~m}$ to $24.70 \mathrm{~m}$ (19\%).

The geometric fitting presented in this work (concerning orthogonal distances) is different from the fitting used by Burša (concerning radial distances). The two methods give the same results for a spherical body or quite similar results for a nearly spherical one, like the Earth. Also, the geometric fitting has the added advantage of a straightforward geometric interpretation of the residuals and the a-posteriori variance factor, something that is difficult to achieve with the algebraic fitting.

We should note that, the procedure to derive the ellipsoidal parameters from the polynomial coefficients (subsection 2.4) is simpler than the corresponding method presented in Bektas (2015). However, it is important to compare the performance of the geometric fitting presented here with the corresponding fitting presented in Bektas.

A further study of the problem of estimating the triaxiality parameters of the Earth could involve other minimization conditions, such as the gravity anomalies (gravimetric method) or the deflections of the vertical. Finally, using the parameters of the triaxial ellipsoid one could also study the differences between this surface and the currently adopted oblate spheroid, as well as their effects, not only regarding the geoid heights but other geometric entities (e.g. geodesic lines) or dynamical characteristics of the Earth.

\section{References}

Barthelmes F., 2014. Global Models. In: Grafarend E. (Ed.) Encyclopedia of Geodesy, Springer International Publishing. https://doi.org/10.1007/978-3-319-02370-0_43-2

Bektas S., 2015. Least squares fitting of ellipsoid using orthogonal distances. Bol Ciênc Geod 21, 329-339. https://doi.org/10.1590/S1982-21702015000200019

Bektas S., 2014. Orthogonal distance from an ellipsoid. Bol Ciênc Geod 20, 970-983. https://doi.org/10.1590/S198221702014000400053

Burša M., 1972. Fundamental geodetic parameters of the earth's figure and the structure of the earth's gravity field derived from satellite data. Stud Geophys Geod 16, 10-29. https://doi.org/10.1007/BF01614229 
Burša M., 1971. On the triaxiality of the earth on the basis of satellite data. Stud Geophys Geod 15, 228-240. https://doi.org/10.1007/BF01589239

Burša M., 1970. Best-fitting tri-axial earth ellipsoid parameters derived from satellite observations. Stud Geophys Geod 14, 1-9. https://doi.org/10.1007/BF02585546

Burša M. and Fialová V., 1993. Parameters of the Earth's tri-axial level ellipsoid. Stud Geophys Geod 37, 1-13. https://doi.org/10.1007/BF01613918

Burša M. and Šíma Z., 1980. Tri-axiality of the Earth, the Moon and Mars. Stud Geophys Geod 24, 211-217. https://doi.org/10.1007/BF01634133

Chen W. and Shen W. B., 2010. New estimates of the inertia tensor and rotation of the triaxial nonrigid Earth. J Geophys Res 115, B12419. https://doi.org/10.1029/2009JB007094

Dermanis A., 2017. Fitting Analytical Surfaces to Points: General Approaches and Applications to Ellipsoid Fitting. In: Georgoula O., Papadopoulou M., Rossikopoulos D., Spatalas S., Fotiou A. (Eds.), Living with GIS, In honour of the memory of Professor Ioannis Paraschakis, AUTh, Thessaloniki, Greece, 81-106.

Eitschberger B., 1978. Ein Geodätisches Weltdatum aus Terrestrischen und Satellitendaten. DGK, Reihe C, Heft 245, München.

GMT 6: Wessel P., Luis J. F., Uieda L., Scharroo R., Wobbe F., Smith W. H. F. and Tian D., 2019. The Generic Mapping Tools version 6. Geochemistry, Geophysics, Geosystems, 20, 5556-5564. https://doi.org/10.1029/2019GC008515

Heiskanen W. A., 1962. Is the Earth a triaxial ellipsoid?. J Geophys Res 67, 321-327. https://doi.org/10.1029/JZ067i001p00321

Hirvonen R. A., 1971. Adjustment by least squares in geodesy and photogrammetry. Ungar, New York.

Karimi R., Ardalan A. A. and Farahani S. V., 2017. The size, shape and orientation of the asteroid Vesta based on data from the Dawn mission. Earth and Planetary Science Letters 475, 71-82. https://doi.org/10.1016/j.epsl.2017.07.033

Karimi R., Ardalan A. A. and Farahani S. V., 2016. Reference surfaces of the planet Mercury from MESSENGER. Icarus 264, 239-245. https://doi.org/10.1016/j.icarus.2015.09.035

Kvas A., Mayer-Gürr T., Krauss S., Brockmann J. M., Schubert T., Schuh W.-D., Pail R., Gruber T., Jäggi A. and Meyer U., 2019. The satellite-only gravity field model G0C006s. GFZ Data Services. http://doi.org/10.5880/ICGEM.2019.002

Ligas M., 2012. Two modified algorithms to transform Cartesian to geodetic coordinates on a triaxial ellipsoid. Stud Geophys Geod 56, 993-1006. https://doi.org/10.1007/s11200-0119017-5

Liu H. S. and Chao B. F., 1991. The Earth's equatorial principal axes and moments of inertia. Geophysical Journal International 106, 699-702. https://doi.org/10.1111/j.1365-246X.1991.tb06341.x

Lemoine F. G., Kenyon S. C., Factor J. K., Trimmer R. G., Pavlis N. K., Chinn D. S., Cox C. M., Klosko S. M., Luthcke S. B., Torrence M. H., Wang Y. M., Williamson R. G., Pavlis E. C., Rapp R. H. and Olson T. R., 1998. The Development of the Joint NASA GSFC and the National Imagery and Mapping Agency (NIMA) Geopotential Model EGM96. NASA/TP-1998-206861, NASA Goddard Space Flight Center, Greenbelt, Maryland.

Lundquist C. A. and Veis G., 1966. Geodetic Parameters for a 1966 Smithsonian Institution Standard Earth. Smithsonian Astrophysical Observatory, Special Report No. 200, Cambridge, Massachusetts.
Mikhail E. M., (with contributions by Ackermann F.), 1976. Observations and least squares. IEP - A Dun - Donnelley Publisher, New York.

NIMA, 2000. Department of Defense World Geodetic System 1984 $\left(3^{\text {rd }}\right)$, US National Imagery and Mapping Agency, Technical Report, 8350.2.

Pavlis N. K., Holmes S. A., Kenyon S. C. and Factor J. K., 2012. The development and evaluation of the Earth Gravitational Model 2008 (EGM2008). J Geophys Res 117, B04406. https://doi.org/10.1029/2011JB008916

Panou G. and Korakitis R., 2019. Cartesian to geodetic coordinates conversion on a triaxial ellipsoid using the bisection method. Preprint, https://www.researchgate.net/publication/ 333904072_Cartesian_to_geodetic_coordinates_conversion_ on_a_triaxial_ellipsoid_using_the_bisection_method

Rapp R. H. and Cruz J. Y., 1986. Spherical Harmonic Expansions of the Earth's Gravitational Potential to Degree 360 Using 30' Mean Anomalies. The Ohio State University, Department of Geodetic Science and Surveying, Report No. 376, Columbus, Ohio.

Tserklevych A. L., Zaiats O. S. and Shylo Y., 2016. Approximation of the physical surface of the Earth by biaxial and triaxial ellipsoid. Geodynamics 20, 40-49.

Wagner C. A., Lerch F. J., Brownd J. E. and Richardson J. A., 1977. Improvement in the geopotential derived from satellite and surface data (GEM 7 and 8). J Geophys Res 82, 901-914. https://doi.org/10.1029/JB082i005p00901 